MATHEMATICS OF COMPUTATION

Volume 67, Number 222, April 1998, Pages 797-814

S $0025-5718(98) 00931-4$

\title{
A CONSTRUCTIVE THEORY OF TRIPLE AND QUINTUPLE PRODUCT IDENTITIES OF THE SECOND DEGREE
}

\author{
RICHARD BLECKSMITH, JOHN BRILLHART, AND IRVING GERST
}

Dedicated to the memory of our wonderful friend and colleague, Irving Gerst

\begin{abstract}
The groundwork for a theory of quadratic identities involving the classical triple and quintuple products is layed. The approach is through the study and use of affine maps that act on indexing lattices associated with the terms (double sums) in the given identity. The terms of the identity are found to be connected by the invariant of a ternary quadratic form.
\end{abstract}

\section{INTRODUCTION}

In this paper we begin to lay the groundwork of a theory of identities whose terms have the form $x^{n}$ times $T_{i} T_{j}, T_{i} Q_{j}$, and $Q_{i} Q_{j}$. Here the letters " $T_{i}, T_{j}$ " and " $Q_{i}, Q_{j}$ " denote respectively one of the four $T$-functions (derived from the Jacobi triple product expansion)

$$
T_{2 \delta+\epsilon}(k, l ; x)=T_{2 \delta+\epsilon}(k, l) \stackrel{\text { def }}{=} \sum_{-\infty}^{\infty}(-1)^{\delta \frac{n(n+1)}{2}+\epsilon n} x^{k n^{2}+l n}
$$

and one of the four $Q$-functions (derived from the quintuple product expansion)

$$
\begin{aligned}
Q_{2 \delta+\epsilon}(k, l ; x) & =Q_{2 \delta+\epsilon}(k, l) \\
& \stackrel{\text { def }}{=} \sum_{-\infty}^{\infty}(-1)^{\delta \frac{n(n+1)}{2}+\epsilon n} x^{\frac{n(3 n+1)}{2} k}\left(x^{-3 n l}-(-1)^{\delta+\epsilon} x^{(3 n+1) l}\right),
\end{aligned}
$$

where $\delta, \epsilon \in\{0,1\}$. For simplicity, we write $T^{2}, T Q$, and $Q^{2}$ for $T_{i} T_{j}, T_{i} Q_{j}$, and $Q_{i} Q_{j}$ respectively, and refer to identities with terms of this kind as "quadratic" identities. We also write $T(k, l)$ for $T_{0}(k, l)$.

The main concerns of this theory are the construction and classification of identities that are quadratic in $T$ and $Q$ and the development of a general method for proving them. One of the goals of the theory is a complete classification of such identities and the discovery of multi-parameter formulas from which the identities in the classes can be derived. In [2, Theorem 1] we gave a formula of sufficient generality to establish the identities of that paper. It is not as yet clear, however, just what role that particular formula will play in the general classification problem.

Received by the editor May 15, 1996 and, in revised form, December 11, 1996.

1991 Mathematics Subject Classification. Primary 11F11.

Key words and phrases. Quadratic identities, triple product, quintuple product, invariant.

(C)1998 American Mathematical Society 
In this work, we take as our goal the construction of a proof of an identity we have already given two proofs of, viz.

$$
T\left(\frac{21}{2}, \frac{7}{2}\right) T\left(\frac{3}{2}, \frac{3}{2}\right)-x^{2} T\left(\frac{21}{2}, \frac{21}{2}\right) T\left(\frac{3}{2}, \frac{1}{2}\right)=2 T_{1}(21,7) T_{1}(3,1) .
$$

Our purpose in giving a third proof of (1.3) is not that (1.3) is of any particular interest beyond its original use in proving a certain $(\bmod 2)$ congruence in [1], but rather that it can be used to explicate the methods and ideas we wish to present here.

The two earlier proofs of (1.3) employed a general expansion formula to express the identity as a $T^{2}$ equation all of whose terms have the form $x^{\alpha_{n}} T\left(k_{1}, l_{1 n}\right) T\left(k_{2}, l_{2 n}\right)$, where the parameters $k_{1}$ and $k_{2}$ are the same for each term. Such equations are called "balanced" at the particular pair $\left(k_{1}, k_{2}\right)$. The resulting equation, which was balanced at the $k$-pair $(96,672)$, was then established by a summation technique called an "expansion of zero." (See [1] for an explanation of these techniques and the relevant terminology.)

In Section 2 of this paper, we expand (1.3) into a $T^{2}$ equation with 16 terms on a side, balanced at the smallest possible $k$-pair $(24,168)$. This 32 term identity is found to be the sum of four sub-identities, each with four terms on a side. Since these four $T^{2}$ equations are similar, we will develop the ideas of this paper using only the first of these as a model, proving all four identities in a uniform way in the last section of the paper.

The initial step toward proving this first identity comes from writing each of its eight terms as a doubly-indexed sum:

$$
x^{\alpha} T\left(k_{1}, l_{1}\right) T\left(k_{2}, l_{2}\right)=\sum_{(i, j) \in \mathbb{Z}^{2}} x^{k_{1} i^{2}+k_{2} j^{2}+l_{1} i+l_{2} j+\alpha} .
$$

Because each coefficient in such a sum is a 1 , the equality between the two sides of the identity is an assertion that the powers of $x$ on the two sides are the same, so the right side is merely a re-arrangement of the left. Thus, to find a proof of the identity is to find its re-arrangement scheme.

In Section 3 we examine the re-arrangement in the first identity by determining which of the four sums on the right contain the powers of $x$ in each of the four sums on the left. It becomes clear from this examination that the lattice points $(i, j)$ in the plane $\mathbb{Z}^{2}$ for a given sum on the left, whose powers of $x$ are in a particular sum on the right, lie on a lattice, and the four lattices in one plane, that correspond respectively to the four sums on the right, form a partition of that plane. The occurrence of such a simple structure as a lattice in the indexing planes permits a precise description of the re-arrangement as a collection of 1-1 affine maps between the lattices in the four indexing planes on the two sides of the identity.

Sections 4-6 deal with the general theory used to establish the re-arrangement schemes. In Section 4 it is proved that if two lattices in $\mathbb{Z}^{2}$ are to be point-wise associated, a certain affine map with rational coefficients must generally be used.

In Section 5, the nature of an affine map that connects two quadratic polynomials of the form $k_{1} x^{2}+k_{2} y^{2}+l_{1} x+l_{2} y+\alpha$ is derived. (These quadratics are the exponent polynomials in (1.4) whose pair of coefficients $\left(k_{1}, k_{2}\right)$ is the same.) In particular, it is discovered that the determinant of such a map must be \pm 1 and the members of the family of quadratic exponent polynomials that appear in an identity, whose members are linked by such affine maps, are restricted to having coefficients which must satisfy a certain invariant. 
Section 6 contains a synthesis of the analyses in Sections 4 and 5. This synthesis shows how to construct affine maps between the members of the family of exponent polynomials so as to properly re-arrange the powers of $x$ by point-wise connections between the respective lattices in $\mathbb{Z}^{2}$. The result of this synthesis is the useful Theorem 3, which gives three computable conditions under which identities like the four identities under consideration are true.

The paper ends with Section 7 in which the four identities are proved using Theorem 3, but not by just applying this theorem to each of the four identities. Instead, Theorem 3 is used to prove a 3-parameter identity (Theorem 4), which contains the four identities as special cases.

\section{EXPANDING IDENTITY (1.3) INTO A BALANCED $T^{2}$ IDENTITY}

In $[1$, p. 792$]$, we discussed expanding identity (1.3) into an identity balanced at $(24,168)$, the smallest possible $K$-pair one can use for this purpose. (We will use capital $K$ and $L$ in this section as in the expansion formula in [1]. In what follows, however, we will use small $k$ and $l$ for the coefficients in the exponent polynomials.) This pair was not used, however, because the resulting identity could not be proved using expansions of zero, the method of proof discussed in that paper. Equation (1.3) was therefore established by expanding to the larger $k$-pair $(96,672)$. In the present paper, we will use the minimal $K$-pair $(24,168)$ to prove the expanded identity by a re-arrangement method of proof.

Before applying the expansion formula, we must use the Backward Program [1, p. 790] to determine the possible $\left(k_{1}, k_{2}\right)$ pairs and associated parameters $[a, b, m]$ that expand to $K_{1}=24, K_{2}=168$ (or vice versa). The four possibilities $\left(k_{1}, k_{2}\right)$ and their associated parameters $[a, b, m]$ that were output by program Backward are:

$$
\begin{aligned}
& \# 1:\left(\frac{21}{2}, \frac{3}{2}\right),[3,3,16] ; \quad \# 2:(21,3),[1,1,8] ; \\
& \# 3:(3,21),[1,7,8] ; \quad \# 4:(21,3),[7,1,8] .
\end{aligned}
$$

Since there is only one possibility starting with $\left(\frac{21}{2}, \frac{3}{2}\right)$, we expand the two terms on the left side of (1.3) using expansion \#1. The double term on the RHS of (1.3) is expanded as two identical terms, using expansions \#2 and \#4. Transposing negative terms to the other side, and canceling 16 matching terms, we obtain the equation

$$
\sum_{i=1}^{16} A_{i}(x)=\sum_{i=1}^{16} B_{i}(x),
$$

where the $A_{i}(x)$ and $B_{i}(x)$ are listed in Table 1 . It is this equation that we must prove.

If we next compare the powers of $x$ that appear in the $A$ and $B$ series on the two sides of (2.1), we discover the surprising fact that (2.1) is actually the sum of four simpler identities, each with four $A$ terms on the left and four $B$ terms on the right. These sub-identities are listed in Table 2.

That none of these four sub-identities can be split further is due to the fact that there is no other dependency among the $A$ and $B$ power series. We describe this characteristic of an identity by the following terminology.

Definition 1. A balanced $T^{2}$ identity is called reducible if it can be split into the sum of two or more balanced $T^{2}$ identities. Otherwise, it is called irreducible. 
TABLE $1 . A_{i}$ and $B_{i}$ in equation (2.1).

$$
A_{i}(x)=x^{\alpha_{i}} T\left(24, l_{1 i}\right) T\left(168, l_{2 i}\right) \quad B_{i}(x)=x^{\alpha_{i}} T\left(24, l_{1 i}\right) T\left(168, l_{2 i}\right)
$$

\begin{tabular}{|c|rrr|}
\hline$i$ & $\alpha_{i}$ & $l_{1 i}$ & $l_{2 i}$ \\
\hline 1 & 0 & 1 & 21 \\
2 & 2 & 2 & 42 \\
3 & 3 & 10 & 42 \\
4 & 3 & 17 & 21 \\
5 & 4 & 14 & 42 \\
6 & 7 & 22 & 42 \\
7 & 7 & 13 & 63 \\
8 & 9 & 19 & 63 \\
9 & 16 & 5 & 105 \\
10 & 17 & 11 & 105 \\
11 & 23 & 2 & 126 \\
12 & 24 & 10 & 126 \\
13 & 25 & 14 & 126 \\
14 & 28 & 22 & 126 \\
15 & 32 & 7 & 147 \\
16 & 37 & 23 & 147 \\
\hline
\end{tabular}

\begin{tabular}{|c|rrr|}
\hline$i$ & $\alpha_{i}$ & $l_{1 i}$ & $l_{2 i}$ \\
\hline 1 & 0 & 6 & 14 \\
2 & 2 & 9 & 35 \\
3 & 3 & 18 & 14 \\
4 & 3 & 3 & 49 \\
5 & 4 & 21 & 7 \\
6 & 7 & 6 & 70 \\
7 & 9 & 9 & 77 \\
8 & 10 & 18 & 70 \\
9 & 14 & 15 & 91 \\
10 & 14 & 6 & 98 \\
11 & 17 & 18 & 98 \\
12 & 25 & 21 & 119 \\
13 & 28 & 15 & 133 \\
14 & 35 & 6 & 154 \\
15 & 38 & 18 & 154 \\
16 & 38 & 3 & 161 \\
\hline
\end{tabular}

TABLE 2. The four sub-identities.

Identity 1

\begin{tabular}{|c|c|c|c|c|c|c|c|c|c|c|c|}
\hline \# & $\alpha$ & $k_{1}$ & $l_{1}$ & $k_{2}$ & & $\#$ & $\alpha^{\prime}$ & $k_{1}$ & $l_{1}^{\prime}$ & $k_{2}$ & $l_{2}^{\prime}$ \\
\hline 1 & 0 & 24 & 1 & 168 & 21 & 1 & 0 & 24 & 6 & 168 & 14 \\
\hline 7 & 7 & 24 & 13 & 168 & 63 & 6 & 7 & 24 & 6 & 168 & 70 \\
\hline 10 & 17 & 24 & 11 & 168 & 105 & 11 & 17 & 24 & 18 & 168 & 98 \\
\hline 16 & 37 & 24 & 23 & 168 & 147 & 15 & 38 & 24 & 18 & 168 & 154 \\
\hline
\end{tabular}

Identity 2 (with multiplier $x^{2}$ canceled)

\begin{tabular}{|c|rrrrr|}
\hline$\#$ & \multicolumn{1}{|c}{$\alpha$} & $k_{1}$ & $l_{1}$ & \multicolumn{1}{c}{$k_{2}$} & \multicolumn{1}{c|}{$l_{2}$} \\
\hline 2 & 0 & 24 & 2 & 168 & 42 \\
3 & 1 & 24 & 10 & 168 & 42 \\
13 & 23 & 24 & 14 & 168 & 126 \\
14 & 26 & 24 & 22 & 168 & 126 \\
\hline
\end{tabular}

\begin{tabular}{|c|rrrrr|}
\hline$\#$ & \multicolumn{1}{|c}{$\alpha^{\prime}$} & $k_{1}$ & $l_{1}^{\prime}$ & $k_{2}$ & \multicolumn{1}{c|}{$l_{2}^{\prime}$} \\
\hline 2 & 0 & 24 & 9 & 168 & 35 \\
4 & 1 & 24 & 3 & 168 & 49 \\
12 & 23 & 24 & 21 & 168 & 119 \\
13 & 26 & 24 & 15 & 168 & 133 \\
\hline
\end{tabular}

Identity 3 (with multiplier $x^{3}$ canceled)

\begin{tabular}{|c|rrrrr|}
\hline$\#$ & $\alpha$ & $k_{1}$ & $l_{1}$ & $k_{2}$ & \multicolumn{1}{c|}{$l_{2}$} \\
\hline 4 & 0 & 24 & 17 & 168 & 21 \\
8 & 6 & 24 & 19 & 168 & 63 \\
9 & 13 & 24 & 5 & 168 & 105 \\
15 & 29 & 24 & 7 & 168 & 147 \\
\hline
\end{tabular}

\begin{tabular}{|c|rrrrr|}
\hline$\#$ & $\alpha^{\prime}$ & $k_{1}$ & $l_{1}^{\prime}$ & $k_{2}$ & \multicolumn{1}{c|}{$l_{2}^{\prime}$} \\
\hline 3 & 0 & 24 & 18 & 168 & 14 \\
8 & 7 & 24 & 18 & 168 & 70 \\
10 & 11 & 24 & 6 & 168 & 98 \\
14 & 32 & 24 & 6 & 168 & 154 \\
\hline
\end{tabular}

Identity 4 (with multiplier $x^{4}$ canceled)

\begin{tabular}{|c|rrrrr|}
\hline$\#$ & \multicolumn{1}{|c}{$\alpha$} & $k_{1}$ & \multicolumn{1}{c|}{$l_{1}$} & \multicolumn{1}{c|}{$k_{2}$} & \multicolumn{1}{c|}{$l_{2}$} \\
\hline 5 & 0 & 24 & 14 & 168 & 42 \\
6 & 3 & 24 & 22 & 168 & 42 \\
11 & 19 & 24 & 2 & 168 & 126 \\
12 & 20 & 24 & 10 & 168 & 126 \\
\hline
\end{tabular}

\begin{tabular}{|c|rcrcr|}
\hline$\#$ & $\alpha^{\prime}$ & $k_{1}$ & $l_{1}^{\prime}$ & $k_{2}$ & \multicolumn{1}{c|}{$l_{2}^{\prime}$} \\
\hline 5 & 0 & 24 & 21 & 168 & 7 \\
7 & 5 & 24 & 9 & 168 & 77 \\
9 & 10 & 24 & 15 & 168 & 91 \\
16 & 34 & 24 & 3 & 168 & 161 \\
\hline
\end{tabular}




\section{THE RE-ARRANGEMENT SCHEMES}

In this section we discuss the idea of the re-arrangement scheme of a balanced $T^{2}$ identity. We will explain this idea by examining the scheme of the first identity in Table 2 (equation (3.1)), which is essentially the same as the schemes of the other three identities in Table 2 . This identity is:

$$
A_{1}(x)+A_{7}(x)+A_{10}(x)+A_{16}(x)=B_{1}(x)+B_{6}(x)+B_{11}(x)+B_{15}(x) .
$$

We begin by writing the eight terms of (3.1) as double sums as in (1.4), so (3.1) becomes

$$
\sum_{r=1}^{4} \sum_{(i, j) \in \mathbb{Z}^{2}} x^{L_{r}(i, j)}=\sum_{s=1}^{4} \sum_{(i, j) \in \mathbb{Z}^{2}} x^{R_{s}(i, j)},
$$

where $L_{r}(i, j)=24 i^{2}+168 j^{2}+l_{1 r} i+l_{2 r} j+\alpha_{r}$ and $R_{s}(i, j)=24 i^{2}+168 j^{2}+l_{1 s}^{\prime} i+$ $l_{2 s}^{\prime} j+\alpha_{s}^{\prime}$. Here the $l_{i r}$ and $l_{i s}$ are respectively $l_{i}$ and $l_{i}^{\prime}$ in Identity 1 in Table 2 . In a few cases we will use the negatives of the values in Table 2, because this gives greater regularity in the resulting geometric lattice patterns that underlie the rearrangements. That such a change is permissible is due to the fact that the indices $i$ and $j$ run over $\mathbb{Z}$.

In this form, it is clear that the equality in (3.1) is an assertion that a power $x^{L_{r}(i, j)}$, evaluated at the point $(i, j)$ in the $r^{\text {th }}$ indexing plane $\mathbb{Z}^{2}$ on the left, will be one of the powers $x^{R_{s}(i, j)}$, evaluated at some corresponding point $\left(i^{\prime}, j^{\prime}\right)$ in the $s^{\text {th }}$ indexing plane $\mathbb{Z}^{2}$ on the right.

It becomes clear after considerable computing that the points $(i, j) \in \mathbb{Z}^{2}$ in the $r^{\text {th }}$ indexing plane, at which $x^{L_{r}(i, j)}$ is a term in the $s^{t h}$ sum on the right, lie on an affine lattice, and that the corresponding points $\left(i^{\prime}, j^{\prime}\right) \in \mathbb{Z}^{2}$ lie on an affine lattice in the $s^{\text {th }}$ indexing plane as well.

Definition 2. A $\underline{2 \text {-dimensional, }}$ ( $\underline{\text { affine }}$ ) lattice is a set of points

$$
\mathcal{L}=\left\{\bar{x} \in \mathbb{Z}^{2}: \bar{x}=n_{1} \bar{b}_{1}+n_{2} \bar{b}_{2}+\bar{x}_{0}, \quad \forall n_{1}, n_{2} \in \mathbb{Z}\right\},
$$

where $\bar{b}_{1}, \bar{b}_{2}, \bar{x}_{0} \in \mathbb{Z}^{2}$ and $\bar{b}_{1}, \bar{b}_{2}$ are linearly independent over $\mathbb{Z}$.

To illustrate such lattices, consider the first sum on the left and the second sum on the right, which have the exponents $L_{1}(i, j)=24 i^{2}+168 j^{2}-i+21 j$ and $R_{2}(i, j)=24 i^{2}+168 j^{2}+6 i-70 j+7$ respectively, with two negative coefficients as mentioned after (3.2).

We find that the lattice for the first sum is

$$
\left[\begin{array}{l}
x \\
y
\end{array}\right]=n_{1}\left[\begin{array}{l}
1 \\
1
\end{array}\right]+n_{2}\left[\begin{array}{l}
4 \\
0
\end{array}\right]+\left[\begin{array}{c}
-1 \\
0
\end{array}\right]=\left[\begin{array}{ll}
1 & 4 \\
1 & 0
\end{array}\right]\left[\begin{array}{l}
n_{1} \\
n_{2}
\end{array}\right]+\left[\begin{array}{c}
-1 \\
0
\end{array}\right], \quad \forall n_{1}, n_{2} \in \mathbb{Z},
$$

and the associated lattice for the second sum is

$$
\left[\begin{array}{l}
x^{\prime} \\
y^{\prime}
\end{array}\right]=n_{1}\left[\begin{array}{c}
-1 \\
1
\end{array}\right]+n_{2}\left[\begin{array}{l}
3 \\
1
\end{array}\right]+\left[\begin{array}{c}
-1 \\
0
\end{array}\right]=\left[\begin{array}{cc}
-1 & 3 \\
1 & 1
\end{array}\right]\left[\begin{array}{l}
n_{1} \\
n_{2}
\end{array}\right]+\left[\begin{array}{c}
-1 \\
0
\end{array}\right]
$$

To verify these are corresponding lattices, we note simply that

$$
\begin{aligned}
L_{1}\left(n_{1}+4 n_{2}-1, n_{1}\right) & =R_{2}\left(-n_{1}+3 n_{2}-1, n_{1}+n_{2}\right) \\
& =192 n_{1}^{2}+384 n_{2}^{2}+192 n_{1} n_{2}-28 n_{1}-196 n_{2}+25 .
\end{aligned}
$$


Lattices such as those in (3.3) and (3.4) and equations similar to (3.5) can be worked out for the other 15 pairs of exponents in Identity 1 . It can also be shown that the quartets of lattices in the eight indexing planes that are linked in this way by the sixteen 1-1 lattice maps, consititute a partitioning of the 8 indexing planes. The other 3 identities in Table 2 can be verified in the same way by finding 48 more equations like (3.5).

Instead of taking this tedious and unrevealing approach, we will pursue the present example further and investigate the nature of the direct map that connects the points $\left[\begin{array}{l}x^{\prime} \\ y^{\prime}\end{array}\right]$ to $\left[\begin{array}{l}x \\ y\end{array}\right]$. To do this, solve (3.4) for $\left[\begin{array}{l}n_{1} \\ n_{2}\end{array}\right]$ and substitute the result into (3.3). This gives the vector equation

$$
\left[\begin{array}{l}
x^{\prime} \\
y^{\prime}
\end{array}\right]=\frac{1}{4}\left[\begin{array}{cc}
3 & -7 \\
1 & 3
\end{array}\right]\left[\begin{array}{l}
x \\
y
\end{array}\right]+\frac{1}{4}\left[\begin{array}{c}
-1 \\
1
\end{array}\right] .
$$

The map $\psi$, defined by $\left[\begin{array}{l}x^{\prime} \\ y^{\prime}\end{array}\right]=\psi\left(\left[\begin{array}{l}x \\ y\end{array}\right]\right)=A\left[\begin{array}{l}x \\ y\end{array}\right]+\bar{d}$, where $A=\frac{1}{4}\left[\begin{array}{cc}3 & -7 \\ 1 & 3\end{array}\right]$ and $\bar{d}=\frac{1}{4}\left[\begin{array}{c}-1 \\ 1\end{array}\right]$, can generally be considered as an affine map between two real planes $\mathbb{R}^{2}$, so that the equality $L_{1}(x, y)=R_{2}\left(x^{\prime}, y^{\prime}\right)$ holds between these two-variable, real polynomials. In general, we call a real, affine map $\psi: \mathbb{R}^{2} \rightarrow \mathbb{R}^{2}$ of this kind a global map. The map $\psi$ in (3.6) has the special property that it is also a 1-1 map of the lattice in (3.3) to the lattice in (3.4).

Not all global maps from $\mathbb{Z}^{2}$ to $\mathbb{Z}^{2}$ send lattices to lattices though. For example, the simple translation

$$
L_{1}(x, y)=R_{2}\left(x-\frac{7}{24}, y+\frac{13}{24}\right)
$$

is a global map from $L_{1}$ to $R_{2}$, but one that doesn't send any integer point to another integer point.

Global maps with rational coefficients turn out to be crucial to our understanding of re-arrangement schemes. They show how the quadratic exponent polynomials relate to each other in a balanced identity. Global maps of the right kind also allow us to relate quadratic exponent polynomials to the underlying re-arrangement lattices. In general, we need to have a simple way to find a global map between a pair of lattices in one of the four planes on the two sides of our identity and, vice versa, to determine when a global map induces a correspondence between lattices in the indexing planes.

\section{RAtional MATRICES AND ASsociated LATtice Maps}

In this short section we prove a theorem about lattices and affine maps between them. The maps between two lattices in $\mathbb{Z}^{2}$ will ordinarily have rational rather than integer coefficients.

Theorem 1 (Lattice Mapping Theorem). (a) Let $\psi: \mathbb{R}^{2} \rightarrow \mathbb{R}^{2}$ be a 1-1 affine map with rational coefficients. Let $K(\psi)=\left\{\bar{x} \in \mathbb{Z}^{2}: \psi(\bar{x}) \in \mathbb{Z}^{2}\right\}$. If $K(\psi) \neq \emptyset$, then $K(\psi)$ and $\psi(K(\psi))$ are lattices in $\mathbb{Z}^{2}$. (b) Conversely, if $\mathcal{L}$ and $\mathcal{L}^{\prime}$ are lattices of $\mathbb{Z}^{2}$, then there exists a 1-1 affine map with rational coefficients $\psi: \mathbb{R}^{2} \rightarrow \mathbb{R}^{2}$ such that $K(\psi)=\mathcal{L}$ and $\mathcal{L}^{\prime}=\psi(\mathcal{L})$. 
Proof. (a) Let $\psi(\bar{x})=A \bar{x}+\bar{b}$, where $A$ and $\bar{b}$ have rational entries. Since $K \neq \emptyset$, there exist $\bar{x}_{0}, \bar{y}_{0} \in \mathbb{Z}^{2}$ such that $\bar{y}_{0}=\psi\left(\bar{x}_{0}\right)=A \bar{x}_{0}+\bar{b}$. Subtracting, we get $\psi(\bar{x})=A\left(\bar{x}-\bar{x}_{0}\right)+\bar{y}_{0}$. Setting $\bar{x}^{\prime}=\bar{x}-\bar{x}_{0}$, we have $\psi\left(\bar{x}^{\prime}+\bar{x}_{0}\right)=A \bar{x}^{\prime}+\bar{y}_{0}$. Defining $\psi_{1}\left(\bar{x}^{\prime}\right)=\psi\left(\bar{x}^{\prime}+\bar{x}_{0}\right)-\bar{y}_{0}$, we have that $\psi_{1}\left(\bar{x}^{\prime}\right)=A \bar{x}^{\prime}$.

Consider the set $K\left(\psi_{1}\right)=\left\{\bar{x}^{\prime} \in \mathbb{Z}^{2}: \psi_{1}\left(\bar{x}^{\prime}\right) \in \mathbb{Z}^{2}\right\}$. If $d=L C M$ of the denominators of the entries in $A$, then $\left[\begin{array}{l}d \\ 0\end{array}\right]$ and $\left[\begin{array}{l}0 \\ d\end{array}\right]$ are linearly independent vectors in $K\left(\psi_{1}\right)$, so $K\left(\psi_{1}\right)$ contains at least two distinct vectors. Now, if $\bar{x}_{1}^{\prime}, \bar{x}_{2}^{\prime} \in K\left(\psi_{1}\right)$, then for any $n_{1}, n_{2} \in \mathbb{Z}$,

$$
\psi_{1}\left(n_{1} \bar{x}_{1}^{\prime}+n_{2} \bar{x}_{2}^{\prime}\right)=n_{1} \psi_{1}\left(\bar{x}_{1}^{\prime}\right)+n_{2} \psi_{1}\left(\bar{x}_{2}^{\prime}\right) \in \mathbb{Z}^{2},
$$

so $K\left(\psi_{1}\right)$ is a lattice in $\mathbb{Z}^{2}$, which implies that $K(\psi)=K\left(\psi_{1}\right)+\bar{x}_{0}$ is a lattice in $\mathbb{Z}^{2}$. Since $\psi_{1}$ is a $1-1$, linear map, it follows that $\psi(K(\psi))=\psi_{1}\left(K\left(\psi_{1}\right)\right)+\bar{y}_{0}$ is also a lattice in $\mathbb{Z}^{2}$.

(b) Let $\mathcal{L}=\left\{B\left[\begin{array}{l}n_{1} \\ n_{2}\end{array}\right]+\bar{x}_{0}\right\}$ and $\mathcal{L}^{\prime}=\left\{C\left[\begin{array}{l}n_{1} \\ n_{2}\end{array}\right]+\bar{y}_{0}\right\}$, where the columns of $B=\left[\begin{array}{ll}\bar{b}_{1} & \bar{b}_{2}\end{array}\right]$ and $C=\left[\begin{array}{ll}\bar{c}_{1} & \bar{c}_{2}\end{array}\right]$ are the respective lattice bases. Clearly $B^{-1}$ exists and has rational entries. Let $A=C B^{-1} \in \mathrm{GL}(2, \mathbb{Q})$. Set $\bar{z}_{0}=\bar{y}_{0}-A \bar{x}_{0} \in \mathbb{Q}^{2}$ and let $\psi(\bar{x})=A \bar{x}+\bar{z}_{0}$. Then for each lattice point in $\mathcal{L}$, we have

$$
\psi\left(B\left[\begin{array}{l}
n_{1} \\
n_{2}
\end{array}\right]+\bar{x}_{0}\right)=A\left(B\left[\begin{array}{l}
n_{1} \\
n_{2}
\end{array}\right]+\bar{x}_{0}\right)+\bar{z}_{0}=C\left[\begin{array}{l}
n_{1} \\
n_{2}
\end{array}\right]+\bar{y}_{0} \in \mathcal{L}^{\prime} .
$$

Since det $A \neq 0, \psi$ is $1-1$, which proves (b).

Remarks. 1. A simple example in which $K(\psi)=\emptyset$ is the map with $A=I$ and $\bar{b}=\frac{1}{2}\left[\begin{array}{l}1 \\ 1\end{array}\right]$.

2. We have phrased Theorem 1 to be used in only the two-dimensional case and only where $\psi$ is $1-1$. It is clear that more general versions of this theorem hold for $n$-dimensional lattices or when $\psi$ is not necessarily $1-1$. It is worth noting that even when $\psi: R^{n} \rightarrow R^{n}$ is not $1-1$, a nonempty $K(\psi)$ will nonetheless have dimension $n$.

3. When an affine map is given, such as

$$
\psi\left(\left[\begin{array}{l}
x \\
y
\end{array}\right]\right)=\frac{1}{m}\left(\left[\begin{array}{ll}
a & b \\
c & d
\end{array}\right]\left[\begin{array}{l}
x \\
y
\end{array}\right]+\left[\begin{array}{l}
e \\
f
\end{array}\right]\right)
$$

where the small letters are integers and $m>0$ is the least common denominator of the component fractions, we can find bases for the lattices $\mathcal{L}$ and $\mathcal{L}^{\prime}$ (see [4]) by solving the system

$$
\left[\begin{array}{ll}
a & b \\
c & d
\end{array}\right]\left[\begin{array}{l}
x \\
y
\end{array}\right] \equiv\left[\begin{array}{l}
-e \\
-f
\end{array}\right] \quad(\bmod m)
$$

The result in the following example, which illustrates Remark 3, will be needed later in the paper.

Example. Let $m$ be a positive integer. Find all integer points $\left[\begin{array}{l}x \\ y\end{array}\right]$ so that $\frac{1}{m}\left[\begin{array}{cc}m-1 & 1-2 m \\ 1 & m-1\end{array}\right]\left[\begin{array}{l}x \\ y\end{array}\right]$ lies in $\mathbb{Z}^{2}$. As in linear algebra, this homogeneous system has the particular solution $\left[\begin{array}{l}0 \\ 0\end{array}\right]$. To find all solutions, reduce the coefficient 
matrix to echelon form, working modulo $m$ :

$$
\left[\begin{array}{cc}
m-1 & 1-2 m \\
1 & m-1
\end{array}\right] \rightarrow\left[\begin{array}{cc}
1 & -1 \\
-1 & 1
\end{array}\right] \rightarrow\left[\begin{array}{cc}
1 & -1 \\
0 & 0
\end{array}\right]
$$

We thus get the single congruence $x \equiv y(\bmod m)$, or equivalently, $x=y+t m$. Setting $n_{1}=y$ and $n_{2}=t$, we obtain the general solution

$$
\left[\begin{array}{l}
x \\
y
\end{array}\right]=n_{1}\left[\begin{array}{l}
1 \\
1
\end{array}\right]+n_{2}\left[\begin{array}{c}
m \\
0
\end{array}\right] .
$$

\section{The GLOBAL MAPS}

The quadratic polynomials that are the exponents in an irreducible, balanced $T^{2}$ equation form an interesting, close-knit family in that the pair of coefficients of their quadratic terms is the same throughout the family ("balanced" quadratics) and the collection of integer values (with repeats) of the quadratics on the left side of the identity — produced by letting the pair of variables in each quadratic range over $\mathbb{Z}^{2}$ — is the same as the collection of values produced in the same way on the right. It seems reasonable under these circumstances to suspect that any two quadratics in such a family might well be connected by an affine map. The next theorem gives necessary and sufficient conditions that specify the form of such an affine map.

Theorem 2 (Global Mapping Theorem). Let $L(x, y)=k_{1} x^{2}+k_{2} y^{2}+l_{1} x+l_{2} y+\alpha$ and $R(x, y)=k_{1} x^{2}+k_{2} y^{2}+l_{1}^{\prime} x+l_{2}^{\prime} y+\alpha^{\prime}$ be in $\mathbb{R}[x, y]$, where $k_{1}, k_{2}>0$ and $k_{2}=k k_{1}$. If $A=\left[\begin{array}{ll}a & b \\ c & d\end{array}\right]$ is a real matrix and $\left[\begin{array}{l}e \\ f\end{array}\right]$ is a real vector, then the vector equation

$$
L\left(\left[\begin{array}{l}
x \\
y
\end{array}\right]\right)=R\left(A\left[\begin{array}{l}
x \\
y
\end{array}\right]+\left[\begin{array}{l}
e \\
f
\end{array}\right]\right)
$$

holds if and only if the following six conditions are satisfied:

$$
\begin{aligned}
& \operatorname{det} A=\epsilon \text {, where } \epsilon= \pm 1 \text {, } \\
& d=\epsilon a, \\
& b=-\epsilon k c \text {, } \\
& a^{2}+k c^{2}=1 \text {, } \\
& {\left[\begin{array}{l}
e \\
f
\end{array}\right]=A\left[\begin{array}{c}
\frac{l_{1}}{2 k_{1}} \\
\frac{l_{2}}{2 k_{2}}
\end{array}\right]-\left[\begin{array}{c}
\frac{l_{1}^{\prime}}{2 k_{1}} \\
\frac{l_{2}^{\prime}}{2 k_{2}}
\end{array}\right],} \\
& \alpha^{\prime}=\alpha+\frac{1}{4}\left[\frac{\left(l_{1}^{\prime}\right)^{2}-l_{1}^{2}}{k_{1}}+\frac{\left(l_{2}^{\prime}\right)^{2}-l_{2}^{2}}{k_{2}}\right] \text {. }
\end{aligned}
$$

Proof. $(\Longrightarrow)$ We have from (5.1) that

$$
\begin{aligned}
k_{1} x^{2}+k_{2} y^{2}+l_{1} x+l_{2} y+\alpha= & k_{1}(a x+b y+e)^{2}+k_{2}(c x+d y+f)^{2} \\
& +l_{1}^{\prime}(a x+b y+e)+l_{2}^{\prime}(c x+d y+f)+\alpha^{\prime} .
\end{aligned}
$$

Equating coefficients of corresponding powers of $x$ and $y$ and canceling common factors gives

$$
a^{2}+k c^{2}=1
$$




$$
\begin{gathered}
b^{2}+k d^{2}=k, \\
a b+k c d=0, \\
l_{1}=2 k_{1} a e+2 k_{2} c f+l_{1}^{\prime} a+l_{2}^{\prime} c,
\end{gathered}
$$

and

$$
l_{2}=2 k_{1} b e+2 k_{2} d f+l_{1}^{\prime} b+l_{2}^{\prime} d .
$$

If $a=0$, then $(5.8)-(5.10)$ give $c^{2}=\frac{1}{k}, d=0$, and $b^{2}=k$. These values satisfy $(5.2)-(5.5)$.

Next assume that $a \neq 0$.

(5.2): Substituting $(5.8)-(5.10)$ in the familiar identity $\left(a^{2}+k c^{2}\right)\left(b^{2}+k d^{2}\right)=$

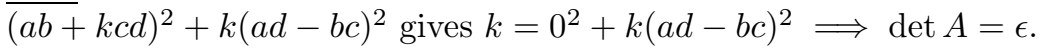

(5.3): Rewriting (5.8) and (5.9) as

$$
\left\{\begin{array}{l}
\left(a^{2}-1\right)+c^{2} k=0 \\
b^{2}+\left(d^{2}-1\right) k=0
\end{array}\right.
$$

we find that

$$
\left|\begin{array}{cc}
a^{2}-1 & c^{2} \\
b^{2} & d^{2}-1
\end{array}\right|=0
$$

Thus,

$$
a^{2}+d^{2}-1=a^{2} d^{2}-b^{2} c^{2}=(a d-b c)(a d+b c)=\epsilon(a d+b c) .
$$

Adding $1=\epsilon(a d-b c)$ in $(5.2)$ gives $d^{2}+a^{2}=2 \epsilon a d$, so $(d-\epsilon a)^{2}=0$, i.e. $d=\epsilon a$.

(5.4): Substituting (5.3) into (5.10) gives $a b+\epsilon k a c=0$. Since $a \neq 0$, then $b=-\epsilon k c$.

(5.5): This is equation (5.8).

$\underline{(5.6)}$ : Combine equations (5.11) and (5.12) as $\left[\begin{array}{l}l_{1} \\ l_{2}\end{array}\right]=2 k_{1}\left[\begin{array}{ll}a & k c \\ b & k d\end{array}\right]\left[\begin{array}{l}e \\ f\end{array}\right]+\left[\begin{array}{ll}a & c \\ b & d\end{array}\right]\left[\begin{array}{l}l_{1}^{\prime} \\ l_{2}^{\prime}\end{array}\right]$.

Multiplying by $\left[\begin{array}{ll}a & b / k \\ c & d / k\end{array}\right]$ gives

$$
\begin{aligned}
A\left[\begin{array}{c}
l_{1} \\
l_{2} / k
\end{array}\right]=\left[\begin{array}{ll}
a & b / k \\
c & d / k
\end{array}\right]\left[\begin{array}{l}
l_{1} \\
l_{2}
\end{array}\right] & =2 k_{1}\left[\begin{array}{ll}
a & b / k \\
c & d / k
\end{array}\right]\left[\begin{array}{ll}
a & k c \\
b & k d
\end{array}\right]\left[\begin{array}{l}
e \\
f
\end{array}\right]+\left[\begin{array}{ll}
a & b / k \\
c & d / k
\end{array}\right]\left[\begin{array}{ll}
a & c \\
b & d
\end{array}\right]\left[\begin{array}{l}
l_{1}^{\prime} \\
l_{2}^{\prime}
\end{array}\right] \\
& =2 k_{1} I\left[\begin{array}{l}
e \\
f
\end{array}\right]+\left[\begin{array}{cc}
1 & 0 \\
0 & 1 / k
\end{array}\right]\left[\begin{array}{l}
l_{1}^{\prime} \\
l_{2}^{\prime}
\end{array}\right]
\end{aligned}
$$

using (5.3) - (5.5). This implies (5.6).

(5.7): Putting

$$
\left[\begin{array}{l}
x \\
y
\end{array}\right]=\left[\begin{array}{l}
-\frac{l_{1}}{2 k_{1}} \\
-\frac{l_{2}}{2 k_{2}}
\end{array}\right]
$$

into (5.1) and using (5.6) gives

$$
L\left(\left[\begin{array}{c}
-\frac{l_{1}}{2 k_{1}} \\
-\frac{l_{2}}{2 k_{2}}
\end{array}\right]\right)=R\left(A\left[\begin{array}{c}
-\frac{l_{1}}{2 k_{1}} \\
-\frac{l_{2}}{2 k_{2}}
\end{array}\right]+\left[\begin{array}{l}
e \\
f
\end{array}\right]\right)=R\left(\left[\begin{array}{c}
-\frac{l_{1}^{\prime}}{2 k_{1}} \\
-\frac{l_{2}^{\prime}}{2 k_{2}}
\end{array}\right]\right) .
$$


It follows from the definitions of $L$ and $R$ in Theorem 2 that

$$
k_{1} \frac{l_{1}^{2}}{4 k_{1}^{2}}+k_{2} \frac{l_{2}^{2}}{4 k_{2}^{2}}+l_{1} \frac{-l_{1}}{2 k_{1}}+l_{2} \frac{-l_{2}}{2 k_{2}}+\alpha=k_{1} \frac{\left(l_{1}^{\prime}\right)^{2}}{4 k_{1}^{2}}+k_{2} \frac{\left(l_{2}^{\prime}\right)^{2}}{4 k_{2}^{2}}+l_{1}^{\prime} \frac{-l_{1}^{\prime}}{2 k_{1}}+l_{2}^{\prime} \frac{-l_{2}^{\prime}}{2 k_{2}}+\alpha^{\prime},
$$

which implies (5.7).

$(\Longleftarrow) \quad$ Let $a$ and $c$ be any real solutions of (5.5) and set $A=\left[\begin{array}{cc}a & -\epsilon k c \\ c & \epsilon a\end{array}\right]$, where $\epsilon \in\{-1,1\}$. Suppose that $\left[\begin{array}{l}e \\ f\end{array}\right]$ is given by (5.6) and that (5.7) holds. A routine calculation shows that (5.1) is satisfied.

Note that (5.7) can be rewritten as

$$
k_{1} l_{2}^{2}+k_{2} l_{1}^{2}-4 k_{1} k_{2} \alpha=k_{1}\left(l_{2}^{\prime}\right)^{2}+k_{2}\left(l_{1}^{\prime}\right)^{2}-4 k_{1} k_{2} \alpha^{\prime} .
$$

This suggests the following definition.

Definition 3. Let $f(x, y)=k_{1} x^{2}+k_{2} y^{2}+l_{1} x+l_{2} y+\alpha$. Then define the invariant $I$ of $f$ to be,

$$
I(f)=k_{1} l_{2}^{2}+k_{2} l_{1}^{2}-4 k_{1} k_{2} \alpha .
$$

Equation (5.13) asserts that $I(L)=I(R)$.

It is worth noting that two quadratic polynomials $f(x, y)$ and $g(x, y)$ are affinely related as in (5.1) if and only if $I(f)=I(g)$. To see this, observe that $(5.1) \Longrightarrow$ $(5.7) \Longleftrightarrow(5.13) \Longleftrightarrow I(f)=I(g)$. On the other hand, $I(f)=I(g) \Longleftrightarrow(5.7)$. We can take $A=I$ and use (5.6) to define $e$ and $f$, which then implies that (5.1) holds.

That such an invariant appears here and takes the form it does is not surprising. Write $f(x, y)$ in homogeneous form:

$$
f(x, y, z)=z^{2} f\left(\frac{x}{z}, \frac{y}{z}\right)=k_{1} x^{2}+k_{2} y^{2}+\alpha z^{2}+l_{1} x z+l_{2} y z .
$$

In general, if

$$
\tilde{f}(x, y, z)=a_{11} x^{2}+a_{22} y^{2}+a_{33} z^{2}+a_{12} x y+a_{13} x z+a_{23} y z=\left[\begin{array}{ll}
x y & z
\end{array}\right] \tilde{A}\left[\begin{array}{l}
x \\
y \\
z
\end{array}\right],
$$

where

$$
\tilde{A}=\left[\begin{array}{ccc}
a_{11} & \frac{a_{12}}{2} & \frac{a_{13}}{2} \\
\frac{a_{12}}{2} & a_{22} & \frac{a_{23}}{2} \\
\frac{a_{13}}{2} & \frac{a_{23}}{2} & a_{33}
\end{array}\right]
$$

then it is well-known that $\operatorname{det} \tilde{A}$ is an invariant. In fact, it is the only fundamental algebraic invariant $[3$, p. 15$]$. When $\tilde{f}(x, y, z)=f(x, y, z)$ in $(5.15)$, determinant $\tilde{A}$ becomes

$$
\left|\begin{array}{ccc}
k_{1} & 0 & \frac{l_{1}}{2} \\
0 & k_{2} & \frac{l_{2}}{2} \\
\frac{l_{1}}{2} & \frac{l_{2}}{2} & \alpha
\end{array}\right|=\alpha k_{1} k_{2}-k_{2} \frac{l_{1}^{2}}{4}-k_{1} \frac{l_{2}^{2}}{4}=-\frac{1}{4} I(f) .
$$

Note that $I(f)=10752$ for all the quadratics $f$ in the four identities in Table 2 , if the powers of $x$ are not canceled. 
Remarks. 1. Observe that $k$ is not required to be an integer in Theorem 2.

2. We have shown in Theorem 2 that if the quadratic exponents are linked by affine maps as in (5.1), then certain conditions must hold. A basic question remains, however, which we put as an (unproved) conjecture:

Conjecture. Any pair of quadratic exponents in an irreducible, balanced $T^{2}$ identity is connected by a global map.

\section{Connecting global maps with lattices}

Our goal in this section is to find lattices $\mathcal{L}$ and $\mathcal{L}^{\prime}$ for $L(x, y)$ and $R(x, y)$ in Theorem 2 such that to each lattice point $(i, j) \in \mathcal{L}$ there corresponds exactly one lattice point $\left(i^{\prime}, j^{\prime}\right) \in \mathcal{L}^{\prime}$ such that $L(i, j)=R\left(i^{\prime}, j^{\prime}\right)$. In other words, we wish to find basis vectors $\bar{b}_{1}, \bar{b}_{2}, \bar{c}_{1}, \bar{c}_{2}$ and displacement vectors $\bar{g}$ and $\bar{g}^{\prime}$ such that

$$
L\left(n_{1} \bar{b}_{1}+n_{2} \bar{b}_{2}+\bar{g}\right)=R\left(n_{1} \bar{c}_{1}+n_{2} \bar{c}_{2}+\bar{g}^{\prime}\right), \quad \forall n_{1}, n_{2} \in \mathbb{Z} .
$$

(It is convenient to use the same coordinates $\left(n_{1}, n_{2}\right)$ for the two lattices.) To find the desired vectors, we employ the matrix $A=\left[\begin{array}{cc}a & -\epsilon k c \\ c & \epsilon a\end{array}\right]$ with rational entries. Now set $a=\frac{\delta}{m}$ and $c=\frac{\gamma}{m}$, where $m$ is the least common denominator of $a$ and $c$. Then,

$$
A=\frac{1}{m} \widehat{A}=\frac{1}{m}\left[\begin{array}{cc}
\delta & -\epsilon k \gamma \\
\gamma & \epsilon \delta
\end{array}\right],
$$

where the integers $\delta$ and $\gamma$ satisfy $\delta^{2}+k \gamma^{2}=m^{2}$.

Next consider the affine map with rational coefficients $\psi\left(\left[\begin{array}{l}x \\ y\end{array}\right]\right)=A\left[\begin{array}{l}x \\ y\end{array}\right]+\left[\begin{array}{l}e \\ f\end{array}\right]$, where $e$ and $f$ are computed in (5.6). By Theorem 1, $\psi$ maps a lattice $\mathcal{L}$ onto a lattice $\mathcal{L}^{\prime}$ if and only if there is a single point $\left[\begin{array}{l}g \\ h\end{array}\right] \in \mathcal{L} \subset \mathbb{Z}^{2}$ such that

$$
\left[\begin{array}{l}
g^{\prime} \\
h^{\prime}
\end{array}\right]=\psi\left(\left[\begin{array}{l}
g \\
h
\end{array}\right]\right)=A\left[\begin{array}{l}
g \\
h
\end{array}\right]+\left[\begin{array}{l}
e \\
f
\end{array}\right] \in \mathcal{L}^{\prime} \subset \mathbb{Z}^{2} .
$$

While $A$ has entries with common denominator $m$, (5.6) shows that $e$ and $f$ have denominators $2 k_{1}$ and $2 k_{2}$ respectively. In order for (6.2) to hold, the rationals $e$ and $f$ must each reduce to a fraction with denominator $m$.

We make two observations. The first is that $k=7$ divides every $l_{2}$ and $l_{2}^{\prime}$ in Table 2. We will assume from now on that $k$ divides $l_{2}$ and $l_{2}^{\prime}$ and we write $l_{2}=k \widehat{l_{2}}$ and $l_{2}^{\prime}=k \widehat{l_{2}^{\prime}}$. The second observation is that $T(k,-l)=T(k, l)$, so we can arbitrarily change the signs of $l_{1}, l_{2}, l_{1}^{\prime}$, and $l_{2}^{\prime}$, if we choose. Thus, to try to make (6.2) hold, we must find $\epsilon_{1}, \epsilon_{2}, \epsilon_{1}^{\prime}, \epsilon_{2}^{\prime} \in\{-1,1\}$, so that

$$
\widehat{A}\left[\begin{array}{l}
\epsilon_{1} l_{1} \\
\epsilon_{2} \widehat{l}_{2}
\end{array}\right] \equiv m\left[\begin{array}{l}
\epsilon_{1}^{\prime} l_{1}^{\prime} \\
\epsilon_{2}^{\prime} \widehat{l}_{2}^{\prime}
\end{array}\right] \quad\left(\bmod 2 k_{1}\right)
$$

When this condition is satisfied, we can write

$$
\left[\begin{array}{l}
e \\
f
\end{array}\right]=\frac{1}{2 k_{1}}\left(\frac{1}{m} \widehat{A}\left[\begin{array}{l}
\epsilon_{1} l_{1} \\
\epsilon_{2} l_{2}
\end{array}\right]-\left[\begin{array}{l}
\epsilon_{1}^{\prime} l_{1}^{\prime} \\
\epsilon_{2}^{\prime} l_{2}^{\prime}
\end{array}\right]\right)=\frac{1}{m}\left[\begin{array}{l}
\hat{e} \\
\widehat{f}
\end{array}\right],
$$


where $\widehat{e}, \widehat{f} \in \mathbb{Z}^{2}$. Our rational map $\psi$ is now

$$
\psi\left(\left[\begin{array}{l}
x \\
y
\end{array}\right]\right)=\frac{1}{m}\left(\widehat{A}\left[\begin{array}{l}
x \\
y
\end{array}\right]+\left[\begin{array}{l}
\widehat{e} \\
\widehat{f}
\end{array}\right]\right) .
$$

We can next examine the system $\bmod m$ as in (4.1) to see if we obtain a solution of (6.2). When we do, we can find an integral matrix $B$ and a vector $\left[\begin{array}{l}g \\ h\end{array}\right] \in \mathbb{Z}^{2}$ such that $\psi\left(B\left[\begin{array}{l}n_{1} \\ n_{2}\end{array}\right]+\left[\begin{array}{l}g \\ h\end{array}\right]\right) \in \mathbb{Z}^{2}$, for each $\left[\begin{array}{l}n_{1} \\ n_{2}\end{array}\right] \in \mathbb{Z}^{2}$. Thus, both the matrix $C=\left[c_{i j}\right]=A B$ and the vector $\left[\begin{array}{l}g^{\prime} \\ h^{\prime}\end{array}\right]$ in (6.2) have integer entries. This produces the two desired lattices, using the columns of the matrices $B$ and $C$ as the basis vectors:

$$
\mathcal{L}:\left[\begin{array}{l}
i \\
j
\end{array}\right]=n_{1}\left[\begin{array}{l}
b_{11} \\
b_{21}
\end{array}\right]+n_{2}\left[\begin{array}{l}
b_{12} \\
b_{22}
\end{array}\right]+\left[\begin{array}{l}
g \\
h
\end{array}\right]
$$

and

$$
\mathcal{L}^{\prime}:\left[\begin{array}{l}
i^{\prime} \\
j^{\prime}
\end{array}\right]=n_{1}\left[\begin{array}{l}
c_{11} \\
c_{21}
\end{array}\right]+n_{2}\left[\begin{array}{l}
c_{12} \\
c_{22}
\end{array}\right]+\left[\begin{array}{l}
g^{\prime} \\
h^{\prime}
\end{array}\right]
$$

so we have

$$
L\left(n_{1}\left[\begin{array}{l}
b_{11} \\
b_{21}
\end{array}\right]+n_{2}\left[\begin{array}{l}
b_{12} \\
b_{22}
\end{array}\right]+\left[\begin{array}{l}
g \\
h
\end{array}\right]\right)=R\left(n_{1}\left[\begin{array}{l}
c_{11} \\
c_{21}
\end{array}\right]+n_{2}\left[\begin{array}{l}
c_{12} \\
c_{22}
\end{array}\right]+\left[\begin{array}{l}
g^{\prime} \\
h^{\prime}
\end{array}\right]\right) .
$$

Before we prove the main theorem (Theorem 3), which deals with identities having the re-arrangement pattern of the identities in Table 2, we will construct a particular global map, related to Identity 1, by choosing a specific solution to (6.2). This will be used in Section 8 where we prove the four identities in Table 2.

When $k$ is an odd integer, we take $m=\frac{k+1}{2}, \delta=\frac{k-1}{2}=m-1, \gamma=1$, and $\epsilon=1$. For these values, $\delta^{2}+\gamma^{2}=m^{2}$, so the matrix $A$ given in (6.1) is a global map. By (4.2), the homogeneous system

$$
\widehat{A}\left[\begin{array}{l}
x \\
y
\end{array}\right]=\left[\begin{array}{cc}
m-1 & 1-2 m \\
1 & m-1
\end{array}\right]\left[\begin{array}{l}
x \\
y
\end{array}\right] \equiv\left[\begin{array}{l}
0 \\
0
\end{array}\right] \quad(\bmod m)
$$

has the general solution $\left[\begin{array}{l}x \\ y\end{array}\right]=n_{1}\left[\begin{array}{l}1 \\ 1\end{array}\right]+n_{2}\left[\begin{array}{c}m \\ 0\end{array}\right]$. Thus, we have

$$
B=\left[\begin{array}{cc}
1 & m \\
1 & 0
\end{array}\right] \quad \text { and } \quad C=A B=\left[\begin{array}{cc}
-1 & m-1 \\
1 & 1
\end{array}\right] \text {. }
$$

The next lemma gives a simple test for determining whether the affine map $A\left[\begin{array}{l}x \\ y\end{array}\right]+\left[\begin{array}{l}e \\ f\end{array}\right]$ maps a point in $\mathbb{Z}^{2}$ to a point in $\mathbb{Z}^{2}$.

Lemma 1. Given two balanced quadratic polynomials $L(x, y)=k_{1} x^{2}+k_{2} y^{2}+l_{1} x+$ $k \widehat{l}_{2} y+\alpha$ and $R(x, y)=k_{1} x^{2}+k_{2} y^{2}+l_{1}^{\prime} x+k \widehat{l}_{2}^{\prime} y+\alpha^{\prime}$, where $k_{1}, k_{2} \in \mathbb{Z}^{+}$and $k=\frac{k_{2}}{k_{1}}$ is an odd integer. Let $A=\frac{1}{m}\left[\begin{array}{cc}m-1 & 1-2 m \\ 1 & m-1\end{array}\right]$, where $m=\frac{k+1}{2}$. Assume for some choice of signs $\epsilon_{1}, \epsilon_{2}, \epsilon_{1}^{\prime}, \epsilon_{2}^{\prime} \in\{1,-1\}$ that

$$
\left[\begin{array}{cc}
1 & -1 \\
1 & m-1
\end{array}\right]\left[\begin{array}{l}
\epsilon_{1} l_{1} \\
\epsilon_{2} \widehat{l}_{2}
\end{array}\right] \equiv\left[\begin{array}{cc}
1 & 1 \\
0 & m
\end{array}\right]\left[\begin{array}{l}
\epsilon_{1}^{\prime} l_{1}^{\prime} \\
\epsilon_{2}^{\prime} \widehat{l}_{2}^{\prime}
\end{array}\right] \quad\left(\bmod 2 k_{1}\right)
$$


Then for $e, f$ defined in (6.4), $\left[\begin{array}{l}g \\ h\end{array}\right]=\left[\begin{array}{c}m e \\ 0\end{array}\right]$ is a vector in $\mathbb{Z}^{2}$ such that $A\left[\begin{array}{l}g \\ h\end{array}\right]+$ $\left[\begin{array}{l}e \\ f\end{array}\right] \in \mathbb{Z}^{2}$.

Proof. To simplify notation, write $\bar{l}=\left[\begin{array}{l}\epsilon_{1} l_{1} \\ \epsilon_{2} \widehat{l}_{2}\end{array}\right]$ and $\quad \bar{l}^{\prime}=\left[\begin{array}{l}\epsilon_{1}^{\prime} l_{1}^{\prime} \\ \epsilon_{2}^{\prime} \widehat{l}_{2}^{\prime}\end{array}\right]$. Multiplying (6.8) by $\left[\begin{array}{cc}m & -1 \\ 0 & 1\end{array}\right]$, we have that

$$
\left[\begin{array}{cc}
m-1 & 1-2 m \\
1 & m-1
\end{array}\right] \bar{l} \equiv\left[\begin{array}{cc}
m & 0 \\
0 & m
\end{array}\right] \bar{l}^{\prime} \Longrightarrow\left[\begin{array}{cc}
m-1 & 1-2 m \\
1 & m-1
\end{array}\right] \bar{l} \equiv m \bar{l}^{\prime}\left(\bmod 2 k_{1}\right),
$$

which is condition (6.3). This implies by (6.4) that

$$
e=\frac{\widehat{e}}{m} \text { and } f=\frac{\widehat{f}}{m}
$$

where $\widehat{e}, \widehat{f} \in \mathbb{Z}$.

We now turn our attention to finding a vector $\left[\begin{array}{l}g \\ h\end{array}\right]$ so that $A\left[\begin{array}{l}g \\ h\end{array}\right]+\left[\begin{array}{l}e \\ f\end{array}\right]$ is an integer. Since all the fractions of this affine map have denominator $m$, this means we must solve the congruence

$$
\widehat{A}\left[\begin{array}{l}
g \\
h
\end{array}\right] \equiv-\left[\begin{array}{l}
\widehat{e} \\
\widehat{f}
\end{array}\right] \quad(\bmod m) .
$$

The augmented matrix for this system reduces $(\bmod m)$ as follows:

$$
\begin{aligned}
{\left[\begin{array}{cc|c}
m-1 & 1-2 m & -\widehat{e} \\
1 & m-1 & -\widehat{f}
\end{array}\right] } & \rightarrow\left[\begin{array}{cc|c}
m-1 & 1-2 m & -\widehat{e} \\
m & -m & -\widehat{e}-\widehat{f}
\end{array}\right] \\
& \rightarrow\left[\begin{array}{cc|c}
1 & -1 & \widehat{e} \\
0 & m & \widehat{e}+\widehat{f}
\end{array}\right] .
\end{aligned}
$$

Thus, the second congruence drops out if and only if $\widehat{e}+\widehat{f} \equiv 0(\bmod m)$. Since by (6.4) and (6.9),

$$
\left[\begin{array}{l}
\widehat{e} \\
\hat{f}
\end{array}\right]=\frac{1}{2 k_{1}}\left(\widehat{A} \bar{l}-m \bar{l}^{\prime}\right)=\frac{1}{2 k_{1}}\left(\left[\begin{array}{cc}
m-1 & 1-2 m \\
1 & m-1
\end{array}\right] \bar{l}-\left[\begin{array}{cc}
m & 0 \\
0 & m
\end{array}\right] \bar{l}^{\prime}\right),
$$

then on adding the first row to the second row, we see the second row is

$$
\widehat{e}+\widehat{f}=\frac{1}{2 k_{1}}\left(\left[\begin{array}{ll}
m & -m
\end{array}\right] \bar{l}-\left[\begin{array}{ll}
m & m
\end{array}\right] \bar{l}^{\prime}\right)=\frac{m}{2 k_{1}}\left(\left[\begin{array}{ll}
1 & -1
\end{array}\right] \bar{l}-\left[\begin{array}{ll}
1 & 1
\end{array}\right] \overline{l^{\prime}}\right) .
$$

By the condition in the first row of $(6.8), 2 k_{1}$ divides $\left[\begin{array}{ll}1 & -1\end{array}\right] \bar{l}-\left[\begin{array}{ll}1 & 1\end{array}\right] \bar{l}^{\prime}$, so $\widehat{e}+\widehat{f} \equiv 0$ $(\bmod m)$, and the system $(6.10)$ is consistent. The first congruence in the reduced augmented matrix in (6.11) is

$$
\left[\begin{array}{ll}
1 & -1
\end{array}\right]\left[\begin{array}{l}
g \\
h
\end{array}\right] \equiv \widehat{e} \quad(\bmod m)
$$

which clearly has the particular solution $\left[\begin{array}{l}g \\ h\end{array}\right]=\left[\begin{array}{l}\widehat{e} \\ 0\end{array}\right]=\left[\begin{array}{c}m e \\ 0\end{array}\right]$.

In determining how various lattices fit together, the following lemma is useful. 
Lemma 2. Assume the hypothesis of Lemma 1. Let $\left[\begin{array}{c}m_{1} \\ n_{1}\end{array}\right],\left[\begin{array}{c}m_{2} \\ n_{2}\end{array}\right] \in \mathbb{Z}^{2}$ be such that $\left[\begin{array}{c}m_{1}^{\prime} \\ n_{1}^{\prime}\end{array}\right]=A\left[\begin{array}{c}m_{1} \\ n_{1}\end{array}\right]+\left[\begin{array}{l}e \\ f\end{array}\right]$ and $\left[\begin{array}{c}m_{2}^{\prime} \\ n_{2}^{\prime}\end{array}\right]=A\left[\begin{array}{c}m_{2} \\ n_{2}\end{array}\right]+\left[\begin{array}{l}e \\ f\end{array}\right]$ are in $\mathbb{Z}^{2}$. Then $m_{1}^{\prime}+n_{1}^{\prime} \equiv m_{2}^{\prime}+n_{2}^{\prime}$ $(\bmod m)$.

Proof. Subtracting the two vector equations gives

$$
\left[\begin{array}{c}
m_{1}^{\prime}-m_{2}^{\prime} \\
n_{1}^{\prime}-n_{2}^{\prime}
\end{array}\right]=A\left[\begin{array}{c}
m_{1}-m_{2} \\
n_{1}-n_{2}
\end{array}\right]=\frac{1}{m}\left[\begin{array}{cc}
m-1 & 1-2 m \\
1 & m-1
\end{array}\right]\left[\begin{array}{c}
m_{1}-m_{2} \\
n_{1}-n_{2}
\end{array}\right] .
$$

Multiplying this equation by the elementary matrix $\left[\begin{array}{ll}1 & 0 \\ 1 & 1\end{array}\right]$ and equating the second components gives

so

$$
m_{1}^{\prime}-m_{2}^{\prime}+n_{1}^{\prime}-n_{2}^{\prime}=\frac{1}{m}\left[\begin{array}{ll}
m & -m
\end{array}\right]\left[\begin{array}{c}
m_{1}-m_{2} \\
n_{1}-n_{2}
\end{array}\right]=m_{1}-m_{2}-\left(n_{1}-n_{2}\right),
$$

$$
\left(m_{1}^{\prime}+n_{1}^{\prime}\right)-\left(m_{2}^{\prime}+n_{2}^{\prime}\right)=\left(m_{1}-n_{1}\right)-\left(m_{2}-n_{2}\right) .
$$

On the other hand, in the first equation, since by hypothesis and (6.9)

$$
A\left[\begin{array}{c}
m_{1} \\
n_{1}
\end{array}\right]+\left[\begin{array}{l}
e \\
f
\end{array}\right]=\frac{1}{m}\left[\begin{array}{cc}
m-1 & 1-2 m \\
1 & m-1
\end{array}\right]\left[\begin{array}{c}
m_{1} \\
n_{1}
\end{array}\right]+\frac{1}{m}\left[\begin{array}{l}
\widehat{e} \\
\hat{f}
\end{array}\right] \in \mathbb{Z}^{2},
$$

we have that the first component is an integer, which is to say $(m-1) m_{1}+$ $(1-2 m) n_{1}+\widehat{e} \equiv 0(\bmod m)$, or reducing, $m_{1}-n_{1} \equiv \widehat{e}(\bmod m)$. Using the second equation, we find in a similar way that $m_{2}-n_{2} \equiv \widehat{e}(\bmod m)$. Subtracting, we obtain that $\left(m_{1}-n_{1}\right)-\left(m_{2}-n_{2}\right) \equiv 0(\bmod m)$. Combining this with $(6.12)$ completes the proof.

Theorem 3. Given:

(a) $k_{1}, k_{2} \in \mathbb{Z}^{+}$, where $k=\frac{k_{2}}{k_{1}}$ is an odd integer,

(b) $A=\frac{1}{m}\left[\begin{array}{cc}m-1 & 1-2 m \\ 1 & m-1\end{array}\right]$, where $m=\frac{k+1}{2}$,

(c) Two families of quadratic polynomials in $\mathbb{Z}[x, y]$ :

$$
L_{r}(x, y)=k_{1} x^{2}+k_{2} y^{2}+l_{1 r} x+k \widehat{l}_{2 r} y+\alpha_{r}, \quad 1 \leq r \leq m,
$$

and

$$
R_{s}(x, y)=k_{1} x^{2}+k_{2} y^{2}+l_{1 s}^{\prime} x+k \widehat{l}_{2 s}^{\prime} y+\alpha_{s}^{\prime}, \quad 1 \leq s \leq m .
$$

Assume the following three conditions are satisfied:

I. Invariant Condition.

$$
I\left(L_{r}\right)=I\left(R_{s}\right), \quad 1 \leq r, s \leq m ;
$$

II. Lattice Condition. For some choice of $\epsilon_{1 r}, \epsilon_{2 r}, \epsilon_{1 s}^{\prime}, \epsilon_{2 s}^{\prime} \in\{-1,1\}$,

$$
\left[\begin{array}{cc}
1 & -1 \\
1 & m-1
\end{array}\right]\left[\begin{array}{c}
\epsilon_{1 r} l_{1 r} \\
\epsilon_{2 r} \widehat{l}_{2 r}
\end{array}\right] \equiv\left[\begin{array}{cc}
1 & 1 \\
0 & m
\end{array}\right]\left[\begin{array}{c}
\epsilon_{1 s}^{\prime} l_{1 s}^{\prime} \\
\epsilon_{2 s}^{\prime} \widehat{l}_{2 s}^{\prime}
\end{array}\right] \quad\left(\bmod 2 k_{1}\right), \quad 1 \leq r, s \leq m
$$

and

III. Synthesis Condition. For the same choice of $\epsilon$ 's as in Condition II, and $r, s, 1 \leq r, s \leq m$, the sets

$$
\left\{m e_{r 1}, \ldots, m e_{r m}\right\} \quad \text { and } \quad\left\{g_{1 s}^{\prime}+h_{1 s}^{\prime}, \ldots, g_{m s}^{\prime}+h_{m s}^{\prime}\right\}
$$


are complete residue systems modulo $m$, where

$$
\left[\begin{array}{c}
e_{r s} \\
f_{r s}
\end{array}\right]=\frac{1}{2 k_{1}}\left(A\left[\begin{array}{l}
\epsilon_{1 r} l_{1 r} \\
\epsilon_{2 r} \widehat{l}_{2 r}
\end{array}\right]-\left[\begin{array}{l}
\epsilon_{1 s}^{\prime} l_{1 s}^{\prime} \\
\epsilon_{2 s}^{\prime} \widehat{l}_{2 s}^{\prime}
\end{array}\right]\right)
$$

and

$$
\left[\begin{array}{l}
g_{r s}^{\prime} \\
h_{r s}^{\prime}
\end{array}\right]=A\left[\begin{array}{c}
m e_{r s} \\
0
\end{array}\right]+\left[\begin{array}{c}
e_{r s} \\
f_{r s}
\end{array}\right]
$$

Then

$$
\sum_{r=1}^{m} x^{\alpha_{r}} T\left(k_{1}, l_{1 r}\right) T\left(k_{2}, k \widehat{l}_{2 r}\right)=\sum_{s=1}^{m} x^{\alpha_{s}^{\prime}} T\left(k_{1}, l_{1 s}^{\prime}\right) T\left(k_{2}, k \widehat{l}_{2 s}^{\prime}\right) .
$$

Proof. Using Theorem 2, we first show that $\psi_{r s}\left(\left[\begin{array}{l}x \\ y\end{array}\right]\right)=A\left[\begin{array}{l}x \\ y\end{array}\right]+\left[\begin{array}{l}e_{r s} \\ f_{r s}\end{array}\right]$ is a global map such that $L_{r}\left(\left[\begin{array}{l}x \\ y\end{array}\right]\right)=R_{s}\left(\psi_{r s}\left(\left[\begin{array}{l}x \\ y\end{array}\right]\right)\right)$ for the matrix $A$ in (b) and the vector $\left[\begin{array}{l}e_{r s} \\ f_{r s}\end{array}\right]$ in $(6.16)$. That conditions $(5.2)-(5.5)$ hold follows readily from examining $A$ in (b). Condition (5.6) is (6.16), with the factor $k=k_{2} / k_{1}$ canceled, and condition (5.7) is equivalent to the invariant condition (6.13). According to Lemma 1, condition II guarantees that $\left[\begin{array}{c}g_{r s} \\ h_{r s}\end{array}\right]=\left[\begin{array}{c}m e_{r s} \\ 0\end{array}\right]$ is a vector in $\mathbb{Z}^{2}$ such that $\left[\begin{array}{l}g_{r s}^{\prime} \\ h_{r s}^{\prime}\end{array}\right]=\psi_{r s}\left(\left[\begin{array}{l}g_{r s} \\ h_{r s}\end{array}\right]\right)$ is in $\mathbb{Z}^{2}$. By Theorem 1 , the set of points $\left\{\bar{x} \in \mathbb{Z}^{2}: \psi_{r s}(\bar{x}) \in \mathbb{Z}^{2}\right\}$ forms a lattice $\mathcal{L}_{r s}$ whose image under $\psi_{r s}$ is also a lattice $\mathcal{L}_{r s}^{\prime}$. We have worked out the general bases for the lattices $\mathcal{L}_{r s}$ and $\mathcal{L}_{r s}^{\prime}$ in (6.5) and (6.6), where $B=\left[\begin{array}{ll}b_{11} & b_{12} \\ b_{21} & b_{22}\end{array}\right]=\left[\begin{array}{cc}1 & m \\ 1 & 0\end{array}\right]$ and $C=\left[\begin{array}{ll}c_{11} & c_{12} \\ c_{21} & c_{22}\end{array}\right]=\left[\begin{array}{cc}-1 & m-1 \\ 1 & 1\end{array}\right]$ in $(6.7)$, so that

$$
L_{r}\left(n_{1}\left[\begin{array}{l}
1 \\
1
\end{array}\right]+n_{2}\left[\begin{array}{c}
m \\
0
\end{array}\right]+\left[\begin{array}{l}
g_{r s} \\
h_{r s}
\end{array}\right]\right)=R_{s}\left(n_{1}\left[\begin{array}{c}
-1 \\
1
\end{array}\right]+n_{2}\left[\begin{array}{c}
m-1 \\
1
\end{array}\right]+\left[\begin{array}{l}
g_{r s}^{\prime} \\
h_{r s}^{\prime}
\end{array}\right]\right) .
$$

To complete the proof we must show that for each $r$, the $m$ lattices $\mathcal{L}_{r 1}, \ldots, \mathcal{L}_{r m}$ form a partition of the $r$ th indexing plane on the left and for each $s$, the $m$ lattices $\mathcal{L}_{1 s}^{\prime}, \ldots, \mathcal{L}_{m s}^{\prime}$ form a partition of the $s$ th indexing plane on the right. Let $H=\left\{n_{1}\left[\begin{array}{l}1 \\ 1\end{array}\right]+n_{2}\left[\begin{array}{c}m \\ 0\end{array}\right]: n_{1}, n_{2} \in \mathbb{Z}\right\}$ be the $\mathbb{Z}$-module generated by the vectors $\left[\begin{array}{l}b_{11} \\ b_{21}\end{array}\right]=\left[\begin{array}{l}1 \\ 1\end{array}\right]$ and $\left[\begin{array}{l}b_{12} \\ b_{22}\end{array}\right]=\left[\begin{array}{c}m \\ 0\end{array}\right]$. It is clear that each coset of $H$ has the form $H+\left[\begin{array}{c}t_{i} \\ 0\end{array}\right]$ where the integers $t_{i}$ range over a complete residue system modulo $m$. Hence, $\left[\mathbb{Z}^{2}: H\right]=m$. Each lattice $\mathcal{L}_{r s}$ is the coset $H+\left[\begin{array}{c}g_{r s} \\ h_{r s}\end{array}\right]=H+\left[\begin{array}{c}m e_{r s} \\ 0\end{array}\right]$. The first synthesis condition (6.15) guarantees that the $m \operatorname{cosets} \mathcal{L}_{r 1}, \ldots, \mathcal{L}_{r m}$ are distinct and hence form a partition of the $r$ th indexing plane $\mathbb{Z}^{2}$. In the same way, we examine the lattices $\mathcal{L}_{r s}^{\prime}$ on the right. These are all cosets of the $\mathbb{Z}$-module $H^{\prime}=\left\{n_{1}\left[\begin{array}{c}-1 \\ 1\end{array}\right]+n_{2}\left[\begin{array}{c}m-1 \\ 1\end{array}\right]: n_{1}, n_{2} \in \mathbb{Z}\right\}$. As before, it is easy to see that $\left[\mathbb{Z}^{2}: H^{\prime}\right]=m$. Since each lattice $\mathcal{L}_{r s}^{\prime}=\psi_{r s}\left(\mathcal{L}_{r s}\right)$, it follows from Lemma 2 that 
for each $\left[\begin{array}{l}x^{\prime} \\ y^{\prime}\end{array}\right]$ in $\mathcal{L}_{r s}^{\prime}$, the sum $x^{\prime}+y^{\prime}$ is congruent to $g_{r s}^{\prime}+h_{r s}^{\prime}$ modulo $m$. Thus, the lattices $\mathcal{L}_{1 s}^{\prime}, \ldots, \mathcal{L}_{m s}^{\prime}$ are all distinct, since the sum of the coordinates of their coset representatives $g_{1 s}^{\prime}+h_{1 s}^{\prime}, \ldots, g_{m s}^{\prime}+h_{m s}^{\prime}$ are distinct $(\bmod m)$ by the second synthesis condition (6.15). Thus, these $m$ lattices partition the $s$ th indexing plane $\mathbb{Z}^{2}$ on the right.

Note. In Theorem 3, the invariant condition guarantees that the quadratic exponents $L_{r}(x, y)$ and $R_{s}(x, y)$ are affinely related by the function $\psi_{r s}$. Also, the lattice condition produces the 1-1 re-arrangement correspondence in (6.19), using the lattices $\mathcal{L}_{r s}$ and $\mathcal{L}_{r s}^{\prime}$ derived from the global map $\psi_{r s}$. Finally, the two synthesis conditions show that these lattices fit together exactly to fill out their planes.

\section{A general PARAMETRIC IDENTIty}

In Theorem 3, we have an effective means for proving the four identities in Table 2. Rather than doing this directly, we will use Theorem 3 to prove a 3 -parameter identity which contains each of the four identities as a special case.

Theorem 4. Let $k_{1}, k_{2} \in \mathbb{Z}^{+}$such that $k=\frac{k_{2}}{k_{1}} \geq 3$ is an odd integer. Let $m=$ $\frac{k+1}{2}$ and suppose $m \mid 2 k_{1}$. If $e, f \in \frac{1}{2} \mathbb{Z}$ such that $e+f \in \mathbb{Z}$, then

$$
\left.\sum_{n=1}^{m} x^{\alpha_{n}} T\left(k_{1}, l_{1 n}\right) T\left(k_{2}, l_{2 n}\right)\right)=\sum_{n=1}^{m} x^{\alpha_{n}} T\left(k_{1}, l_{1 n}^{\prime}\right) T\left(k_{2}, l_{2 n}^{\prime}\right),
$$

where $\alpha_{n}=\frac{2 k_{1}}{m} n^{2}+2 e n$,

$$
\left\{\begin{array} { l } 
{ l _ { 1 n } = \frac { 2 k _ { 1 } } { m } n + e + f , } \\
{ l _ { 2 n } = k ( \frac { 2 k _ { 1 } } { m } n + e ) - f , }
\end{array} \quad \text { and } \quad \left\{\begin{array}{l}
l_{1 n}^{\prime}=\frac{2 k_{1}}{m} n+e-f, \\
l_{2 n}^{\prime}=k\left(\frac{2 k_{1}}{m} n+e\right)+f .
\end{array}\right.\right.
$$

Proof. We will verify the hypotheses of Theorem 3. Part (a) is clearly satisfied. In part (b), we have $A=\frac{1}{m}\left[\begin{array}{cc}m-1 & -(2 m-1) \\ 1 & m-1\end{array}\right]$. The two quadratic families in part (c) of Theorem 3 are $L_{r}$ and $R_{s}$. It is straightforward using (5.14) and the five formulas in (7.2) to verify that $I\left(L_{r}\right)=I\left(R_{s}\right)=2 m k_{1}\left(k e^{2}+f^{2}\right)$ for the $m$ different $L_{r}$ 's and $R_{s}$ 's. This verifies the Invariant Condition.

Trying various possibilities for the signs, we find that we must take $\epsilon_{1 r}=\epsilon_{2 r}=$ $\epsilon_{2 s}^{\prime}=1$ and $\epsilon_{1 s}^{\prime}=-1$ for $1 \leq r, s \leq m$. Inserting these values into (6.14), we find that

$$
\begin{aligned}
& {\left[\begin{array}{cc}
1 & -1 \\
1 & m-1
\end{array}\right]\left[\begin{array}{l}
\frac{2 k_{1}}{m} r+e+f \\
\frac{2 k_{1}}{m} r+e-\frac{f}{k}
\end{array}\right]=\left[\begin{array}{c}
\frac{f(k+1)}{k} \\
2 k_{1} r+m e+\frac{m f}{k}
\end{array}\right] } \\
& \equiv\left[\begin{array}{c}
\frac{f(k+1)}{k} \\
2 k_{1} s+m e+\frac{m f}{k}
\end{array}\right]=\left[\begin{array}{cc}
1 & 1 \\
0 & m
\end{array}\right]\left[\begin{array}{c}
-\left(\frac{2 k_{1}}{m} s+e-f\right) \\
\frac{2 k_{1}}{m} s+e+\frac{f}{k}
\end{array}\right] \quad\left(\bmod 2 k_{1}\right) .
\end{aligned}
$$

This verifies the lattice condition in Theorem 3. 
TABLE 3

\begin{tabular}{|r|rrrrr|}
\hline$r$ & \multicolumn{1}{|c}{$\alpha$} & $k_{1}$ & $l_{1}$ & $k_{2}$ & \multicolumn{1}{c|}{$l_{2}$} \\
\hline 1 & 17 & 24 & 11 & 168 & 105 \\
2 & 37 & 24 & 23 & 168 & 147 \\
3 & 7 & 24 & 13 & 168 & 63 \\
4 & 0 & 24 & 1 & 168 & 21 \\
\hline
\end{tabular}

\begin{tabular}{|r|rrrrr|}
\hline$s$ & $\alpha^{\prime}$ & $k_{1}$ & $l_{1}^{\prime}$ & $k_{2}$ & \multicolumn{1}{c|}{$l_{2}^{\prime}$} \\
\hline 1 & 17 & 24 & 18 & 168 & 98 \\
2 & 38 & 24 & 18 & 168 & 154 \\
3 & 7 & 24 & 6 & 168 & 70 \\
4 & 0 & 24 & 6 & 168 & 14 \\
\hline
\end{tabular}

It remains to verify the synthesis condition. An elaborate, but straightforward, calculation in 3(b) and (6.18) with much simplification, gives that

$$
\begin{aligned}
{\left[\begin{array}{l}
e_{r s} \\
f_{r s}
\end{array}\right]=\frac{1}{2 k_{1}}\left\{\frac{1}{m}\left[\begin{array}{cc}
m-1 & -(2 m-1) \\
1 & m-1
\end{array}\right]\right.} & {\left[\begin{array}{c}
\frac{2 k_{1}}{m} r+e+f \\
\frac{2 k_{1}}{m} r+e-\frac{f}{2 m-1}
\end{array}\right] } \\
& \left.-\left[\begin{array}{c}
-\left(\frac{2 k_{1}}{m} s+e-f\right) \\
\frac{2 k_{1}}{m} s+e+\frac{f}{2 m-1}
\end{array}\right]\right\}=\frac{1}{m}\left[\begin{array}{l}
s-r \\
r-s
\end{array}\right] .
\end{aligned}
$$

From this we calculate

$$
\left[\begin{array}{l}
g_{r s}^{\prime} \\
h_{r s}^{\prime}
\end{array}\right]=\frac{1}{m}\left[\begin{array}{cc}
m-1 & -(2 m-1) \\
1 & m-1
\end{array}\right]\left[\begin{array}{c}
m \frac{s-r}{m} \\
0
\end{array}\right]+\frac{1}{m}\left[\begin{array}{l}
s-r \\
r-s
\end{array}\right]=\left[\begin{array}{c}
s-r \\
0
\end{array}\right] .
$$

Since $m e_{r s}=s-r=g_{r s}^{\prime}+h_{r s}^{\prime}$, it is clear the synthesis condition (6.15) holds. This completes the proof.

We return to the four identities in Table 2 . Consider Identity 1 , where $k_{1}=24$, $k_{2}=168, k=7$, and $m=4$. The global matrix is $A=\frac{1}{4}\left[\begin{array}{cc}3 & -7 \\ 1 & 3\end{array}\right]$. Theorem 4 now specializes to

$$
\begin{aligned}
\sum_{n=1}^{4} x^{12 n^{2}+2 e n} T(24,12 n+e+f) T(168,84 n+7 e-f) & \\
= & \sum_{n=1}^{4} x^{12 n^{2}+2 e n} T(24,12 n+e-f) T(168,84 n+7 e+f) .
\end{aligned}
$$

Putting $(e, f)=\left(\frac{5}{2}, \frac{7}{2}\right)$ in $(7.3)$ and reducing the $T$-functions as in $[1, \mathrm{p}$. 780], we obtain Identity 1 , with terms in Table 3 in an order different from that of Table 2.

We can establish Identities $2-4$ in Table 2 in a similar way by using the parameters $(e, f)=\left(\frac{11}{2},-\frac{7}{2}\right),\left(\frac{1}{2}, \frac{35}{2}\right)$, and $\left(\frac{7}{2}, \frac{35}{2}\right)$ respectively.

We conclude this work by mentioning that the special form of Theorem 3 relates to its use in deriving Theorem 4, which was designed to prove identities whose re-arrangement scheme is that of the identities in Table 2. There are other re-arrangement schemes for balanced quadratic identities that are much more complicated than the type we dealt with here. To prove these, however, will require a more general theorem. We will examine these matters in another paper.

\section{REFERENCES}

1. R. Blecksmith, J. Brillhart, and I. Gerst, On a certain (mod 2) identity and a method of proof by expansion, Math. Comp. 56 (1991), 775-794. MR 91j:11087

2. R. Blecksmith, J. Brillhart, and I. Gerst, A fundamental modular identity and some applications, Math. Comp. 61 (1993), 83-95. MR 94c:11100 
3. E. B. Elliot, An Introduction to the Algebra of Quantics, Second Edition, Chelsea, New York, 1969.

4. H. J. S. Smith, On systems of linear indeterminant equations and congruences, Collected Math. Papers 1 (1965), 367-409.

Department of Mathematics, Northern Illinois University, DeKalb, Illinois 60115

E-mail address: richard@math.niu.edu

Department of Mathematics, University of Arizona, Tucson, Arizona 85721

E-mail address: jdb@math.arizona.edu

Department of Applied Mathematics and Statistics, SUny at Stony Brook, Stony Brook, New York 11794 